\title{
Campylobacter contamination level in houseflies after exposure to materials containing Campylobacter
}

\author{
Nygaard Jensen, Annette; Hald, Birthe
}

Published in:

Journal of Insects as Food and Feed

Link to article, DOI:

10.3920/JIFF2018.0007

Publication date:

2018

Document Version

Early version, also known as pre-print

Link back to DTU Orbit

Citation (APA):

Nygaard Jensen, A., \& Hald, B. (2018). Campylobacter contamination level in houseflies after exposure to materials containing Campylobacter. Journal of Insects as Food and Feed, 4(3), 179-186.

https://doi.org/10.3920/JIFF2018.0007

\section{General rights}

Copyright and moral rights for the publications made accessible in the public portal are retained by the authors and/or other copyright owners and it is a condition of accessing publications that users recognise and abide by the legal requirements associated with these rights.

- Users may download and print one copy of any publication from the public portal for the purpose of private study or research.

- You may not further distribute the material or use it for any profit-making activity or commercial gain

- You may freely distribute the URL identifying the publication in the public portal

If you believe that this document breaches copyright please contact us providing details, and we will remove access to the work immediately and investigate your claim. 


\title{
Campylobacter contamination level in houseflies after exposure to materials containing Campylobacter
}

\author{
A. N. Jensen ${ }^{1 *}$ and B. Hald ${ }^{1}$. \\ National Food Institute, Technical University of Denmark, Kemitorvet, Bygning 202, DK-2800 Kgs. \\ Lyngby, Denmark; anyj@food.dtu.dk
}

Although houseflies have been found to carry Campylobacter jejuni, little is known about the quantitative campylobacter level in naturally contaminated houseflies and their ability to contaminate surfaces. This study aimed to elucidate how houseflies' previous exposure to campylobacter-contaminated material (faeces or liquid) for 1 or $4 \mathrm{~h}$ affects the acquired campylobacter level in houseflies and their contamination potential.

Cups of 250-ml were added $5 \mathrm{~g}$ of chicken faeces or $1 \mathrm{ml}$ liquid and spiked with approximately 3, 4, 5 or $7 \log _{10}$ CFU C. jejuni. Sixteen houseflies were added to each cup. After $1 \mathrm{~h}$ of exposure, four houseflies were removed from the cup for enumeration of campylobacter in each fly by plate spreading. Another four houseflies were transferred onto Abeyta-Hunt-Bark (AHB) agar plates $(9 \mathrm{~cm}$ ) to assess possible contamination of surfaces. After $1 \mathrm{~h}$ on the AHB plate, each fly was tested for level of campylobacter. This procedure was repeated after approx. $4 \mathrm{~h}$ of exposure for the remaining eight houseflies.

The $C$. jejuni acquisition in houseflies increased with exposure dose and was higher after liquid exposure compared with faeces exposure, while there was no significant effect of exposure time ( 1 vs. 4 h). For faeces, 90.0\% ( $n=80)$, 48.4\% $(n=64), 6.3 \%(n=48)$ and $0 \%$ $(n=16)$ of houseflies were campylobacter-positive when exposed to 7, 5, 4, and $3 \log _{10} \mathrm{CFU}$ with a mean $( \pm \mathrm{SE})$ of $2.0 \pm 0.1,0.8 \pm 0.1,0.3 \pm 0.0$ and $0 \log _{10}$ CFU recovered per campylobacter-positive fly, respectively. For liquid, 95.7\% ( $n=47), 91.4 \%(n=47), 20.8 \%$ $(\mathrm{n}=48)$ and $6.3 \%(\mathrm{n}=16)$ of houseflies were campylobacter-positive with a mean of $3.3 \pm 0.2$, $2.0 \pm 0.1,0.8 \pm 0.2$ and $0.3 \pm 0.0 \log _{10}$ CFU. The surface of the AHB plates was only contaminated by houseflies previously exposed to $>4 \log _{10}$, but the $C$. jejuni number found on the AHB surface did not correlate with the number found in the corresponding fly.

\section{Introduction:}

The bacterial load and species diversity varies between different insect species, where houseflies (Musca domestica) are found to harbor a broad range of species including hostspecies specific bacterial taxa (Bahrndorff et al. 2017; Gupta et al. 2012; Gill et al. 2017; Junqueria et al. 2017). Houseflies feed on decaying organic matter, carcasses and faeces, a feeding behavior implying a substantial exposure to various bacteria incl. human pathogens. Among human pathogens detected in houseflies are Campylobacter spp. incl. C. jejuni, which is the most common cause of foodborne gastrointestinal infections in humans and therefore are flies hypothesized to play a role in transmission of Campylobacter (Ekdahl et al. 2005; Nichols, 2005). The reported Campylobacter spp. prevalence in flies is between $0.2 \%$ and 11\% depending on season and environment (Hald et al. 2004, 2008; Hansson et al. 2007; Royden et al. 2016; Szalanski et al. 2004). The Campylobacter spp. contaminated flies are shown to be a possible transmission vehicle for introduction of Campylobacter spp. from the environment into poultry facilities, as the Campylobacter spp. prevalence were reduced in poultry flocks where fly screens prevented flies physically from entering the poultry facility (Hald et al. 2007, Bahrndorff et al. 2013). Under experimental conditions, Shane et al. (1985) also found that houseflies became contaminated with $C$. jejuni when confined in an isolator with $C$. jejuni infected chicks, and following, these houseflies transmitted $C$. jejuni to a new batch of specific-pathogen-free (SPF) chicks. However, it is uncertain if this $C$. jejuni transmission to chicks occurs indirectly due to houseflies' contamination of the environment 
via excreta and regurgitates or via contact to feet and external body structures, or directly from ingestion of contaminated houseflies. Also, little is known about the specific mechanisms of Campylobacter spp. contamination of houseflies under natural conditions and the houseflies' ability to transmit Campylobacter spp. afterwards. An experimental campylobacter infection model in houseflies indicates that contaminated houseflies turn campylobacter negative within $24 \mathrm{~h}$ after ingestion and that houseflies mainly serve as a mechanical vector without amplification of Campylobacter spp. (Gill et al. 2017; Skovgaard et al. 2011). This is contrary to e.g. Escherichia coli and Enterococcus faecalis, which seem to proliferate in houseflies (Doud and Zurek, 2012; Kobayashi et al. 1999). Attempts have been made to assess the location of bacteria in houseflies after exposure. Shane et al. (1985) found that the recovery of $C$. jejuni was $69 \%$ from viscera and $19 \%$ from feet and ventral surface of the body ( $\mathrm{n}=32$ ) after exposure to a broth containing $2.8 \times 10^{8} \mathrm{CFU} / \mathrm{ml}$. Gill et al. (2017) found that vomitus of inoculated houseflies was $C$. jejuni positive $4 \mathrm{~h}$ but not $8 \mathrm{~h}$ after ingestion of $2.1 \times 10^{6}$ CFU. They also found that $40 \%$ of rinse samples $(n=5)$ from the inside of $1.5 \mathrm{ml}$ Eppendorf tubes containing excreta and vomitus from an inoculated housefly was positive within $1 \mathrm{~h}$ and up to $4 \mathrm{~h}$ after ingestion but not after $8 \mathrm{~h}$. For a better understanding of the Campylobacter spp. infection potential and contamination risk from houseflies, this study aimed to elucidate the quantitative level of $C$. jejuni acquired in houseflies after exposure to contaminated material (natural infection) and also houseflies' ability to contaminate surfaces following. The effect of exposure dose, duration of exposure and type of contaminated material was addressed.

\section{Methods:}

Rearing of houseflies.

The Musca domestica (houseflies) used in this study was obtained from a Danish dairy cattle farm in 1989 and reared continuously under laboratory conditions at $25^{\circ} \mathrm{C}$ and $80 \%$ relative humidity. Eggs were laid and developed in a medium consisting of wheat bran (24.6\%), alfalfa (12.3\%), yeast (0.26\%), malt (0.9\%) and tap water (61.6\%). Adult houseflies were fed on tap water and a mix of sugar (1/3) and skimmed milk powder (2/3).

\section{Preparation of C.jejuni suspension.}

The C. jejuni strain used in this study, SC181, originates from poultry (Bang et al. 2003). For preparation of $C$. jejuni stock suspensions, cells were harvested in $1 \mathrm{ml}$ Brain Heart Infusion (BHI) broth from blood agar (BA) plates (5\% v/v calf blood) with $C$. jejuni subcultures incubated for $16-24 \mathrm{~h}$ at $42^{\circ} \mathrm{C}$ under microaerobic atmosphere $(6 \% \mathrm{O} 2,7 \% \mathrm{CO} 2,7 \% \mathrm{H} 2$ and $80 \%$ N2). The $C$. jejuni stock suspension was adjusted to an optical density (OD) of 0.600 measured at wavelength $600 \mathrm{~nm}(\mathrm{OD} 600 \mathrm{~nm})$ in a UV-mini 1240 spectrophotometer (Shimadzu Europe GmbH, Duisburg, Germany) to reach approximately 9 $\log _{10}$ CFU ml ${ }^{-1}$. Subsequently, C. jejuni suspensions of specified cell concentrations were made by appropriate dilution of the stock suspension in BHI broth.

For all C. jejuni stock suspensions, $\mathrm{CFU} \mathrm{ml^{-1 }}$ was estimated from 10 -fold dilution series in $\mathrm{BHI}$ broth, plated onto BA agar plates and incubated in microaerobic atmosphere at $42^{\circ} \mathrm{C}$ for approximately $48 \mathrm{~h}$ before colony counting

\section{Experimental design.}

The quantitative Campylobacter uptake in houseflies after exposure to different contamination levels in faeces or liquid material was estimated. Houseflies were placed in a 250-ml cup containing a) $5 \mathrm{~g}$ of chicken faeces spiked with 1-ml C. jejuni suspension or b) a lid from a $50 \mathrm{ml}$ CELLSTAR ${ }^{\circledR}$ Polypropylene Tube (Greiner Bio-One GmbH, Frickenhausen, Germany) added 1-ml C. jejuni suspension and covered with a metal grid to avoid drowning of the houseflies. The added 1-ml C. jejuni suspension was adjusted to 
specific concentrations of approximately 3, 4, 5 or $7 \mathrm{Log}_{10} \mathrm{CFU} \mathrm{ml}{ }^{-1}$. Sixteen houseflies were anesthetized with $\mathrm{CO}_{2}$ and placed in each cup covered with a cotton mesh cloth to allow aeration. After approximately $1 \mathrm{~h}$ of exposure to the contaminated material, four houseflies were removed from the cup for enumeration of $C$. jejuni in each fly. Another four single houseflies were each transferred to an Abeyta-Hunt-Bark (AHB) agar plate (9 cm Petri dish) with a sterile tweezer and left on the surface of the agar for $1 \mathrm{~h}$ before enumeration of $C$. jejuni in each fly. The AHB plates were following incubated at $42^{\circ} \mathrm{C}$ under microaerobic atmosphere for $48 \mathrm{~h}$ before visual inspection of $C$. jejuni contamination of the plates. After approximately $4 \mathrm{~h}$ of exposure, the procedure described above was repeated for the remaining eight flies in the cup. This experimental setup was replicated as follows for the different exposure doses, for faeces; $3 \log _{10} C F U(n=1), 4 \log _{10}$ CFU ( $\left.n=3\right), 5 \log _{10} C F U(n=4)$ and 7 $\log _{10}$ CFU (n=5) and for liquid; $3 \log _{10}$ CFU (n=1), $4 \log _{10}$ CFU (n=3), $5 \log _{10}$ CFU (n=3) and $7 \log _{10} \mathrm{CFU}(\mathrm{n}=3)$. Consequently, the total number of houseflies analyzed for each type of material (faeces or liquid) and exposure dose was as follows: Faeces; $3 \log _{10}$ CFU ( $n=16$ ), $4 \log _{10}$ CFU (n=48), $5 \log _{10}$ CFU (n=64) and $7 \log _{10}$ CFU ( $\left.n=80\right)$ and for liquid; $3 \log _{10}$ CFU ( $n=16), 4 \log _{10}$ CFU ( $\left.n=48\right), 5 \log _{10}$ CFU ( $\left.n=48\right)$ and $7 \log _{10}$ CFU ( $\left.n=48\right)$. In total, Campylobacter uptake was estimated for 208 houseflies exposed to contaminated faeces and for 158 houseflies exposed to contaminated liquid (2 houseflies were omitted from analysis). The houseflies' ability to contaminate the surface of AHB plates was assessed for half of the houseflies, i.e. 184.

Enumeration of C. jejuni in houseflies.

At the time of analysis, each cup was flushed with $\mathrm{CO}_{2}$ and placed on ice to immobilize the houseflies before transfer of individual houseflies to a sterile Eppendorf tube with a sterile tweezer. Likewise, the lid of the AHB agar plates was lifted carefully in one side to allow flushing with $\mathrm{CO}_{2}$ before transfer of the fly to an Eppendorf tube. Each fly was added $200 \mu \mathrm{l}$ BHI and crushed with a sterile pellet pestle of polypropylene (Kimble ${ }^{\mathrm{TM}}$ Kontes $^{\mathrm{TM}}$, SigmaAldrich Denmark A/S. Copenhagen, DK) before vortexing. Ten-fold dilutions of the fly homogenate (in duplicate) were spot inoculated ( $3 \times 15 \mu \mathrm{l}$ spots) onto Abeyta-Hunt-Bark (AHB) agar plates. Additionally, for each experiment, three single non-exposed houseflies were included as negative controls. Each fly was added $200 \mu \mathrm{l} \mathrm{BHI}$ and after homogenization the suspension was plated onto AHB.

All agar plates were incubated at $42^{\circ} \mathrm{C}$ under microaerobic atmosphere for approximately $48 \mathrm{~h}$ before counting and calculation of CFU recovered per fly and visual inspection of negative control plates.

\section{Statistical analysis.}

All statistical analyses were performed with GraphPad Prism, 5.0 (GraphPad Software Inc., La Jolla, CA, US). Generally, CFU recovered per fly was transformed by $\log _{10}$ to approximate Normality of data, but not all data passed the Shapiro-Wilk normality test $(P<0.05)$, so non-parametric one way analysis of variance (Kruskal-Wallis) has been undertaken for analyzing the effect of exposure length ( $1 \mathrm{~h}$ vs. $4 \mathrm{~h}$ ) and level ( $\left.\log _{10} \mathrm{CFU}\right)$ using 0.05 as level of significance. To see if the contamination level on AHB plates depended on the houseflies' preceding exposure dose, the proportions of AHB positive plates for 5 $\log _{10}$ CFU and $7 \log _{10}$ CFU were compared by Fischer's exact test for contaminated faeces as well as liquid.

It was tested if the campylobacter contamination observed on an AHB plate was correlated to the number of campylobacter recovered from the corresponding fly by Spearman's rank 
correlation coefficient, non-parametric. This was tested for 36 AHB campylobacter-positive plates (negative AHB plates were excluded from the analysis).

\section{Results:}

Quantitative level of campylobacter in houseflies after exposure

For houseflies exposed to either $C$. jejuni contaminated faeces or liquid, an increased length of exposure from $1 \mathrm{~h}$ to $4 \mathrm{~h}$ did not increase the campylobacter numbers found in the houseflies (analysis by Kruskal-Wallis) as shown in figure 1 and figure 2.

Figure 1. The number of Campylobacter jejuni recovered from positive houseflies (mean $\log _{10}$ CFU and S.E.) after exposure to contaminated faeces for $1 \mathrm{~h}$ (white bars) or $4 \mathrm{~h}$ (black bars).

Figure 2. The number of Campylobacter jejuni recovered from positive houseflies (mean $\log _{10}$ CFU and S.E.) after exposure to contaminated liquid for $1 \mathrm{~h}$ (white bars) or $4 \mathrm{~h}$ (black bars).

Consequently, the $C$. jejuni acquisition level in houseflies after $1 \mathrm{~h}$ and $4 \mathrm{~h}$ of exposure has been pooled, resulting in $90.0 \%(n=80), 48.4 \%(n=64), 6.3 \%(n=48)$ and $0 \%(n=16)$

campylobacter-positive houseflies after exposure to faeces containing approximately 7, 5, 4, and $3 \log _{10} \mathrm{CFU}$, respectively. The mean $( \pm \mathrm{SE}) \mathrm{CFU}$ recovered per campylobacter-positive fly was $2.0 \pm 0.1,0.8 \pm 0.1,0.3 \pm 0.0$ and $0 \log _{10} \mathrm{CFU}$ for exposure levels estimated to be mean ( \pm SE) 7.3 $\pm 0.1,5.4 \pm 0.1,4.3 \pm 0.1$ and $3.4 \pm 0.0 \log _{10}$ CFU respectively. A maximum of 3.6 $\log _{10}$ CFU was found in individual houseflies.

For houseflies exposed to campylobacter contaminated liquid, 95.7\% ( $n=47), 91.4 \%(n=47)$, $20.8 \%(n=48)$ and $6.3 \%(n=16)$ of houseflies were campylobacter-positive when exposed to mean $\left( \pm \mathrm{SE}\right.$ ) $7.2 \pm 0.2,5.2 \pm 0.2,4.6 \pm 0.0$ and $3.6 \pm 0.0 \mathrm{Log}_{10} \mathrm{CFU}$ with a mean $( \pm \mathrm{SE})$ of $3.3 \pm 0.2$, $2.0 \pm 0.1,0.8 \pm 0.2$ and $0.3 \pm 0.0 \log _{10}$ CFU recovered per campylobacter-positive fly. A maximum of $4.8 \log _{10}$ CFU was found in individual houseflies. No C. jejuni was found in the negative control houseflies.

The $C$. jejuni recovery from the houseflies decreased with reduced levels of exposure and at 3 $\log _{10}$ CFU only 1 fly was positive $(n=16)$ after exposure to contaminated liquid. Although the exposure dose in both faeces and liquid overall affected the $C$. jejuni acquisition level by houseflies significantly (Kruskal-Wallis, $P<0.0001$ ), there was no significant difference between an exposure dose of 4 and $5 \log _{10}$ CFU (Dunn's Multiple Comparison Test). A comparison between faeces and liquid contaminated at similar levels also showed a higher acquisition level from liquid than from faeces for 7 and $5 \log _{10} \mathrm{CFU}$, while no significant difference was obtained for 4 and $3 \log _{10}$ CFU.

\section{Houseflies' contamination of surfaces after exposure}

C. jejuni surface contamination of AHB agar plates by houseflies previously exposed to contaminated material was observed for 36 out of 184 plates (19.6\%) and the AHB plates were only contaminated by houseflies previously exposed to at least $5 \log _{10}$ CFU as shown in Table 1. Most AHB plates became contaminated by houseflies previously exposed to liquid $(45 \%, n=48)$ compared with houseflies exposed to faeces $(19 \% n=72)$. 
Tabel 1. Number of AHB plates contaminated with $C$. jejuni by houseflies left for $1 \mathrm{~h}$ on the plate surface after previous exposure to $C$. jejuni containing faeces or liquid.

\begin{tabular}{cccc}
\hline \multicolumn{4}{c}{ No. C. jejuni contaminated } \\
AHB plates ${ }^{1}$ \\
\hline \multicolumn{4}{c}{ Exposure dose and material ${ }^{2}$} \\
$\log _{10}$ & Faeces & $\log _{10}$ & Liquid \\
CFU & & CFU & \\
\hline $3.4 \pm 0.0$ & $0(n=8)$ & $3.6 \pm 0.0$ & $0(n=8)$ \\
$4.3 \pm 0.1$ & $0(n=24)$ & $4.6 \pm 0.0$ & $0(n=24)$ \\
$5.4 \pm 0.1$ & $1(n=32)$ & $5.2 \pm 0.2$ & $8(n=24)$ \\
$7.3 \pm 0.1$ & $13(n=40)$ & $7.2 \pm 0.2$ & $14(n=24)$ \\
\hline
\end{tabular}

${ }^{1}$ Abeyta-Hunt-Bark (AHB) agar plates $(9 \mathrm{~cm}$ Petri dish), $\mathrm{n}=$ number of tested plates.

${ }^{2}$ Houseflies exposed (1-4 h) to either faeces (5 g) or liquid (1 ml) contaminated with $C$. jejuni at different levels.

Also, the proportion of AHB contaminated plates were lower for houseflies exposed to faeces containing 5.4 $\pm 0.1 \log _{10} \mathrm{CFU} C$. jejuni than houseflies exposed to faeces containing $7.3 \pm 0.1$ $\log _{10}$ CFU ( $P=0.002$, Fischer's exact test). Contrary, for houseflies exposed to contaminated liquid, the exposure dose had no significant effect on the proportion of AHB contaminated plates $(P=0.147$, Fischer's exact test $)$.

Moreover, for the 36 contaminated AHB plates $(n=184)$, the $C$. jejuni $\log _{10}$ CFU observed on the plate (mean $0.8 \pm 0.1 \log _{10} \mathrm{CFU}$ and maximum $2.2 \log _{10} \mathrm{CFU}$ ) was not correlated (Spearman $\mathrm{r}, P=0.28$ ) to the numbers of $C$. jejuni recovered from the fly contaminating that plate (mean 2.8 $\pm 0.2 \log _{10} \mathrm{CFU}$ ), see Figure 3. Out of the 148 flies that did not contaminate the AHB plates, 66 flies were tested $C$. jejuni positive (mean $1.6 \pm 0.1 \log _{10} \mathrm{CFU}$ ).

Figure 3. The number of Campylobacter jejuni deposited on the surface of 36 contaminated AHB agar plates $(n=184)$ by houseflies previously exposed to $C$. jejuni containing material vs. the number of $C$. jejuni recovered from that respective fly.

\section{Discussion:}

In this study, it was found that the quantitative C. jejuni acquisition in houseflies after exposure to contaminated material depended on the previous exposure dose and type of material. The lack of a significant effect between the lower exposure doses was probably because of too few observations as only campylobacter-positive houseflies were incl. in the analysis. After exposure to $7.3 \pm 0.1 \log _{10} \mathrm{CFU}$ in $5 \mathrm{~g}$ faeces, the acquired $C$. jejuni level was $2.0 \pm 0.1$ in the $90 \%$ of $C$. jejuni positive houseflies. Although the $10 \%$ negative houseflies were found among the houseflies exposed for the shortest time, the current exposure time $(1 \mathrm{~h}$ vs. $4 \mathrm{~h}$ ) did not exhibit a significant effect on the acquired $C$. jejuni level. For shorter exposure times of $10 \mathrm{~s}$ and $30 \mathrm{~s}$, Pace et al. (2017) found that longer exposure times increased the uptake of E. coli O157:H7 and Salmonella enterica serovar Enteritidis, but also that the uptake level differed between the bacterial species. This may indicate that the bacterial uptake in houseflies occurs upon their first meal shortly after exposure (within $30 \mathrm{~s}$ ) and may explain why no difference in acquisition level was observed between $1 \mathrm{~h}$ and $4 \mathrm{~h}$ of exposure in the current study. However, the thirst of houseflies may differ between studies due to various preexperimental conditions influencing their feeding behavior. Still, even the houseflies treated similarly exhibited quite different feeding behavior during the current exposure study, as some houseflies went straight for the contaminated material while others appeared to sit still on the uncontaminated walls of the cups. No accumulation of $C$. jejuni seemed to take place 
over time either as a consequence of feeding frequency or a continuous excretion and/or regurgitation of ingested bacteria. The latter is supported by findings by Gill et al. (2017) that found that vomitus and excreta were $C$. jejuni positive within the first $4 \mathrm{~h}$ after ingestion (inoculation). Also, the excretion interval in houseflies determined by their eosin coloring of filter paper has been calculated to be 6.5-32.6 min depending on sex and egg developmental stage based on the total excretion over $24 \mathrm{~h}$ (Sasaki et al. 2000).

The acquired $C$. jejuni level per fly (mean $2.0 \pm 0.1 \log _{10} \mathrm{CFU}$ ) after exposure to mean $7.3 \pm 0.1 \log _{10}$ CFU in this study indicated a rather low uptake volume from faeces calculated to be $<0.1 \mu \mathrm{l}$. This apparent uptake capacity of the houseflies implies that their chance of becoming campylobacter positive at lower exposure doses would be markedly reduced. However, as up to $3.6 \log _{10}$ CFU was found in individual houseflies (exposed to faeces) it indicated an uptake volume up to $2.5 \mu \mathrm{l}$, which is similar to the $2-4 \mu \mathrm{l}$ reported by Skovgaard et al. (2011). Moreover, another study showed that female and male houseflies differed in their acquisition of Salmonella Typhimurium from manure but not Escherichia coli (Thomson et al. 2017). In the current study, the houseflies were allocated randomly to the cups without determination of sex so it is unsure to what degree the obtained results may have been influenced by the sex of the houseflies. However, regardless of the potential uptake volume, even at a $C$. jejuni exposure dose approximately $2 \log _{10}$ CFU lower, i.e. $5.4 \pm 0.1 \log _{10}$ CFU, nearly half of the houseflies was tested $C$. jejuni positive (48.4\%) with a mean of $0.8 \pm 0.1$ $\log _{10}$ CFU. This indicates that uptake of campylobacter is likely under natural conditions, as livestock animals and particularly poultry may shed up to $8 \log _{10}$ CFU per g faeces (Nielsen, 2002; Jensen et al. 2006; Rosenquist et al. 2006). So although the Campylobacter spp. prevalence in houseflies is found to be low in most studies (Hald et al. 2004, 2008; Hansson et al. 2007; Royden et al. 2016; Szalanski et al. 2004) and inoculated houseflies seem to turn campylobacter negative within $24 \mathrm{~h}$ (Gill et al. 2017; Skovgaard et al. 2011), the readily uptake of $C$. jejuni under natural exposure levels shown in this study supports that houseflies may play a significant role in transmission of Campylobacter. This is further backed up by an observed C. jejuni prevalence up to $50.7 \%$ in houseflies trapped in a chicken flock facility (Rosef and Kapperud, 1983).

When houseflies were exposed to approximately $4 \log _{10}$ CFU it was mainly houseflies exposed to a liquid suspension that became $C$. jejuni positive (20.8\%). The lower $C$. jejuni acquisition in houseflies exposed to contaminated faeces versus liquid suspension may in part reflect that $C$. jejuni in the faeces set-up was 'hidden' in $5 \mathrm{~g}$ of faeces opposed to the presence in $1 \mathrm{ml}$ of suspension in the liquid set-up. It may also reflect that houseflies must regurgitate to liquefy the faeces before ingestion reducing the actual uptake. However, it emphasizes the importance of avoiding puddles of contaminated water in the farm environment as they may enhance the risk of transferring Campylobacter spp. and also other zoonotic pathogens via contaminated houseflies.

The $C$. jejuni contamination of surfaces by houseflies required a previous exposure dose of minimum $5 \log _{10}$ CFU, Table 1. Although the highest proportion of $C$. jejuni contaminated plates derived from houseflies previously exposed to liquid, there seemed to be no correlation between the contamination level found on the agar surface and the $C$. jejuni numbers recovered from the fly contaminating that plate, Figure 3 . Another study showed transfer of $E$. coli O157:H7 onto spinach leaves tested $18 \mathrm{~h}$ after entry of houseflies that had been exposed to either inoculated manure or a lawn of bacteria in a Petri dish (Talley et al. 2009). Also, only $50 \%$ of the leaves tested E. coli O157:H7 positive when houseflies were exposed to manure reflecting a natural contamination level opposed to a bacterial lawn resulting in $100 \%$ positive leaves. This emphasizes the importance of assessing the effect of different levels of 
exposure in naturally contaminated materials for a better understanding of the C. jejuni transfer potential by houseflies. Further, that the experimental set-up needs careful consideration for proper interpretation of implications. Also indicated by a preliminary study (data not shown) where agar surfaces became heavily campylobacter contaminated by houseflies, most likely because the houseflies had been placed directly on contaminated faeces more or less enforcing contamination and therefore not reflecting natural exposure conditions.

The observed campylobacter uptake in houseflies may also be of relevance in relation to rearing of fly larvae for feed purposes, as the adult flies will be involved in the reproductive cycles although not eaten themselves. However, a study by Bahrndorff et al. (2016) showed that while campylobacter was transmitted between larvae and pupal stages no campylobacter was transmitted further to the adults. This may indicate that 'recycling of infections' between stages can be prevented, but eggs from infected adults should be tested to clarify this.

In conclusion, the $C$. jejuni acquisition level in houseflies depended on the previous level of exposure and occurred down to $4 \log _{10} \mathrm{CFU}$ per $5 \mathrm{~g}$ faeces, which indicates a strong potential for contamination of houseflies in a natural farm setting. Consecutive surface contamination was only observed for houseflies exposed to at least $5 \log _{10}$ but did not correlate to the $C$. jejuni numbers recovered from the houseflies.

\section{Acknowledgement}

This study was supported by the Emida ERA-Net project CamChain "Biology and control of Campylobacter in the chicken supply chain. J. nr. 3405-11-0433. We also thank Steen Nordentoft for valuable help establishing the fly culture in our laboratory.

\section{References:}

Bahrndorff, S., Gill, C., Lowenberger, C., Skovgård, H. and Hald, B. 2014. The Effects of Temperature and Innate Immunity on Transmission of Campylobacter jejuni (Campylobacterales: Campylobacteraceae) Between Life Stages of Musca domestica (Diptera: Muscidae). Journal of Medical Entomology 51, 670-677. DOI: http://dx.doi.org/10.1603/ME13220.

Bahrndorff, S., De Jonge, N., Skovgård, H., Nielsen, J.L. 2017. Bacterial communities associated with houseflies (Musca domestica L.) sampled within and between farms. PLoS ONE, 12 (1), art. no. e0169753

Bahrndorff, S., Rangstrup-Christensen, L., Nordentoft, S., Hald, B. 2013. Foodborne disease prevention and broiler chickens with reduced Campylobacter infection. Emerging Infectious Diseases 19: 425-430.

Bang, D.D., Nielsen, E.M., Knudsen, K. and Madsen, M. 2003. A one-year study of campylobacter carriage by individual Danish broiler chickens as the basis for selection of Campylobacter spp. strains for a chicken infection model. Epidemiology and Infection 13: 323-333. DOI:0.1017/S095026880200821X.

Doud, C.W., Zurek, L. 2012. Enterococcus faecalis OG1RF:pMV158 survives and proliferates in the house fly digestive tract. Journal of Medical Entomology 49: 150-155.

Ekdahl, K., Normann, B., Andersson, Y. 2005. Could flies explain the elusive epidemiology of campylobacteriosis? BMC Infectious Diseases 5: 4. DOI: 10.1186/1471-2334-5-11.

Gupta, A.K., Nayduch, D., Verma, P., Shah, B., Ghate, H.V., Patole, M.S., Shouche, Y.S. 2012. Phylogenetic characterization of bacteria in the gut of house flies (Musca domestica L.). FEMS Microbiology Ecology, 79: 581-593. 
Gill, C., Bahrndorff, S., Lowenberger, C. 2017.Campylobacter jejuni in Musca domestica: An examination of survival and transmission potential in light of the innate immune responses of the house flies. Insect Science, 24: 584-598.

Hald, B., Skovgård, H., Pedersen, K., Bunkenborg, H. 2008. Influxed insects as vectors for Campylobacter jejuni and Campylobacter coli in Danish broiler houses. Poultry Science 87: 1428-1434.

Hald, B., Sommer, H.M., Skovgård, H. 2007. Use of fly screens to reduce Campylobacter spp. introduction in broiler houses. Emerging Infectious Diseases 13: 1951-1953.

Hald, B., Skovgård, H., Bang, D.D., Pedersen, K., Dybdahl, J., Jespersen, J.B., Madsen, M. 2004. Flies and Campylobacter infection of broiler flocks. Emerging Infectious Diseases 10: 1490-1492.

Hansson, I., Vågsholm, I., Svensson, L., Olsson Engvall, E. 2007. Correlations between Campylobacter spp. prevalence in the environment and broiler flocks. Journal of Applied Microbiology 103: 640-649.

Jensen, A.N., Dalsgaard, A., Baggesen, D.L., Nielsen, E.M. 2006. The occurrence and characterization of Campylobacter jejuni and C. coli in organic pigs and their outdoor environment. Veterinary Microbiology 116: 96-105.

Junqueira, A.C.M., Ratan, A., Acerbi, E., Drautz-Moses, D.I., Premkrishnan, B.N.V., Costea, P.I., Linz, B., Purbojati, R.W., Paulo, D.F., Gaultier, N.E., Subramanian, P., Hasan, N.A., Colwell, R.R., Bork, P., Azeredo-Espin, A.M.L., Bryant, D.A., Schuster, S.C. 2017. The microbiomes of blowflies and houseflies as bacterial transmission reservoirs. Scientific Reports 7: art. no. 16353 .

Kobayashi, M., Sasaki, T., Saito, N., Tamura, K., Suzuki, K., Watanabe, H., Agui, N. 1999. Houseflies: Not simple mechanical vectors of enterohemorrhagic Escherichia coli O157:H7. American Journal of Tropical Medicine and Hygiene 61: 625-629.

Nichols, G.L. 2005. Fly transmission of Campylobacter. Emerging Infectious Diseases, 11: 361-364.

Nielsen, E.M. 2002. Occurrence and strain diversity of thermophilic campylobacters in cattle of different age groups in dairy herds. Letters in Applied Microbiology 35: 85-89.

Pace, R.C., Talley, J.L., Crippen, T.L., Wayadande, A.C. 2017. Filth fly transmission of Escherichia coli O157:H7 and Salmonella enterica to lettuce, Lactuca sativa. Annals of the Entomological Society of America 110: 83-89.

Rosef, O., Kapperud, G. House flies (Musca domestica) as possible vectors of Campylobacter fetus subsp. Jejuni. 1983. Applied and Environmental Microbiology 45: 381-383.

Rosenquist, H., Sommer, H.M., Nielsen, N.L., Christensen, B.B. 2006. The effect of slaughter operations on the contamination of chicken carcasses with thermotolerant Campylobacter. International Journal of Food Microbiology 108: 226-232.

Royden, A., Wedley, A., Merga, J.Y., Rushton, S., Hald, B., Humphrey, T., Williams, N.J. 2016. A role for flies (Diptera) in the transmission of Campylobacter to broilers?

Epidemiology and Infection 144: 3326-3334.

Sasaki, T., Kobayashi, M., Agui, N. 2000. Epidemiological potential of excretion and regurgitation by Musca domestica (Diptera: Muscidae) in the dissemination of Escherichia coli O157: H7 to food. Journal of Medical Entomology 37: 945-949. 
Shane, S.M., Montrose, M.S., Harrington, K.S. 1985.Transmission of Campylobacter jejuni by the housefly (Musca domestica). Avian Diseases 29: 384-391.

Skovgård, H., Kristensen, K., Hald, B. 2011. Retention of Campylobacter (Campylobacterales: Campylobacteraceae) in the house fly (Diptera: Muscidae). Journal of Medical Entomology, 48: 1202-1209.

Szalanski, A.L., Owens, C.B., McKay, T., Steelman, C.D. 2004. Detection of Campylobacter and Escherichia coli O157:H7 from filth flies by polymerase chain reaction. Medical and Veterinary Entomology 18: 241-246.

Talley, J.L., Wayadande, A.C., Wasala, L.P., Gerry, A.C., Fletcher, J., DeSilva, U., Gilliland, S.E. 2009. Association of Escherichia coli O157:H7 with filth flies (Muscidae and Calliphoridae) captured in leafy greens fields and experimental transmission of E. coli O157:H7 to spinach leaves by house flies (diptera: Muscidae). Journal of Food Protection 72:1547-1552.

Thomson, J.L., Yeater, K.M., Zurek, L., Nayduch, D. 2017. Abundance and accumulation of Escherichia coli and Salmonella Typhimurium procured by male and female house flies (diptera: Muscidae) exposed to cattle manure. Annals of the Entomological Society of America 110: 37-44. 


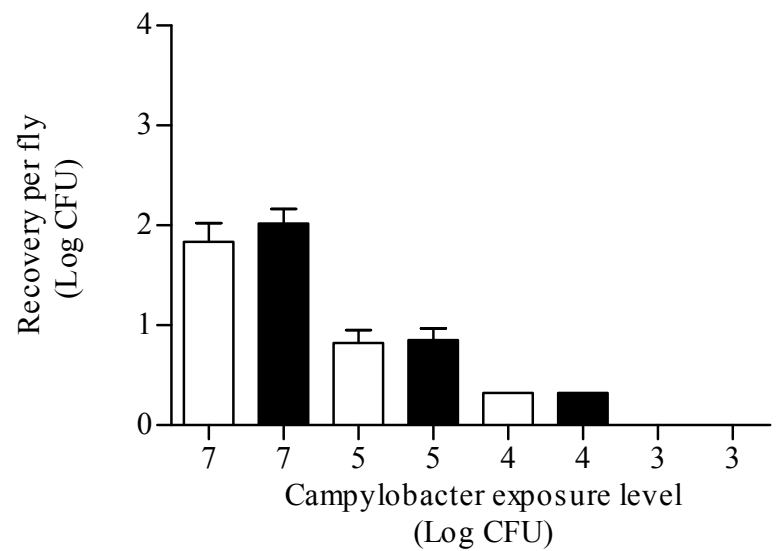




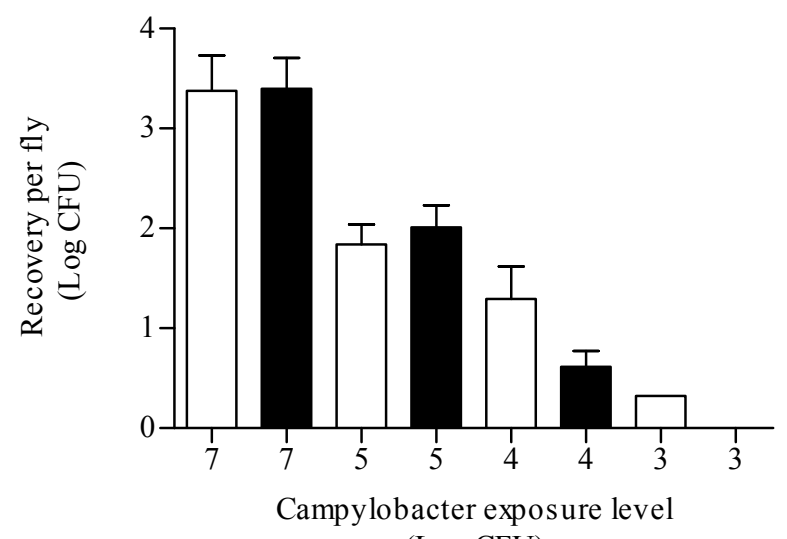

(Log CFU) 


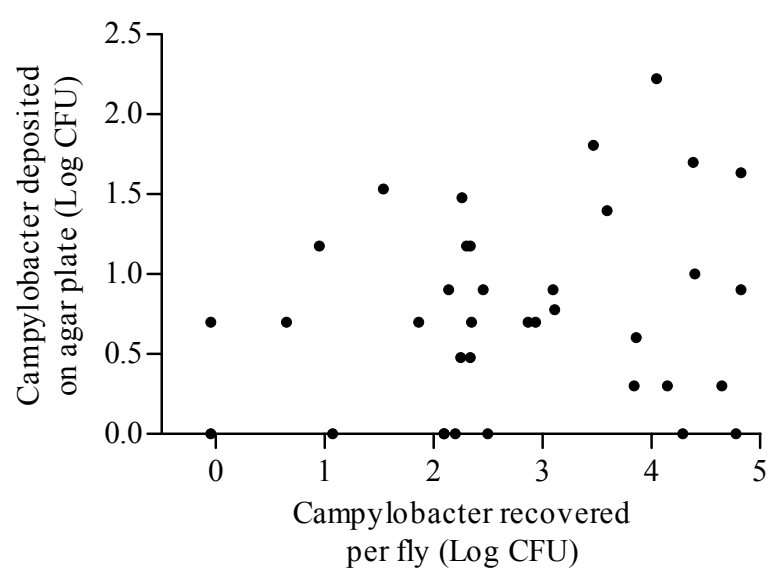

